\title{
Marcadores de consumo alimentar e contexto social de crianças menores de 5 anos de idade
}

\author{
Markers of food consumption and social context of children under \\ 5 years of age
}

\author{
Dixis Figueroa Pedraza' (D), Eduarda Emanuela Silva dos Santos ${ }^{1}$ (D) \\ 'Universidade Estadual da Paraíba (UEPB) - Campina Grande (PB), Brasil.
}

\begin{abstract}
Como citar: Figueroa Pedraza D, Santos EES. Marcadores de consumo alimentar e contexto social de crianças menores de 5 anos de idade. Cad Saúde Colet, 2021;29(2):163-178. https://doi.org/10.1590/1414-462X202129020072
\end{abstract}

\begin{abstract}
Resumo
Introdução: A avaliação do consumo alimentar das crianças é essencial para as medidas de promoção de saúde delas. Objetivo: Avaliar os marcadores do consumo alimentar de crianças menores de 5 anos atendidas na Estratégia Saúde da Família e verificar sua associação com o contexto social. Método: Estudo desenvolvido nos municípios da Paraíba, prioritários para ações voltadas à prevenção da obesidade infantil, com amostra probabilística representativa de 909 crianças (893 estudadas). Resultados: As prevalências de aleitamento materno exclusivo e complementar foram de $40,4 \%$ e 56,8\%, respectivamente; diversidade alimentar, de 18,2\%; consumo de fruta, de 74,9\%; e consumo de verduras e legumes, de 61,2\%. O consumo de alimentos ultraprocessados foi de $51,1 \%$ para crianças < 2 anos e de $25,8 \%$ para crianças de 2-5 anos. A situação socioeconômica associou-se à interrupção do aleitamento materno e ao consumo de fruta; o apoio social, ao aleitamento materno exclusivo, à interrupção do aleitamento materno e ao consumo de fruta; a assistência social, à interrupção do aleitamento materno, ao consumo de alimentos ultraprocessados, à diversidade alimentar e ao consumo das principais refeições; a segurança alimentar e nutricional, à diversidade alimentar e ao consumo de fruta. Conclusão: A utilização dos marcadores do consumo alimentar possibilitou a identificação de práticas alimentares inadequadas e fatores do contexto social associados. Palavras-chave: estratégia saúde da família; criança; consumo de alimentos; vigilância nutricional.
\end{abstract}

\begin{abstract}
Background: The assessment of child food intake is essential for health promotion measures. Objective: To evaluate markers of food consumption in children under 5 years of age assisted at the Family Health Strategy and verify their association with social context. Method: The study was conducted in the municipalities of the state of Paraíba, Brazil, considered as priority for actions aimed to prevent childhood obesity, with a probabilistic and representative sample composed of 909 children (893 studied). Results: The following prevalence rates were observed: exclusive and complementary breastfeeding (40.4 and $56.8 \%$, respectively), food diversity $(18.2 \%)$, fruit consumption $(74.9 \%)$, consumption of vegetables (61.2\%), and consumption of ultra-processed foods by children aged $<2$ and 2-5 years (51.1 and 25.8\%, respectively). The following associations between social context and markers of food intake were found: socioeconomic status - interruption of breastfeeding and fruit consumption; social support - exclusive breastfeeding and interruption of breastfeeding and fruit consumption; social assistance - interruption of breastfeeding, consumption of ultra-processed foods, food diversity, and consumption of main meals; food and nutrition security - food diversity and fruit consumption. Conclusion: The use of markers of food consumption allowed the identification of inadequate food practices and factors associated with social context.
\end{abstract}

Keywords: family health strategy; children; food consumption; nutrition surveillance.

Trabalho realizado em unidades básicas de saúde da família e creches de nove municípios do Estado da Paraíba - Bayeux Cabedelo, Cajazeiras, Mamanguape, Monteiro, Pombal, Queimadas, São Bento, Sousa (PB), Brasil.

Correspondência: Dixis Figueroa Pedraza. E-mail: dixisfigueroa@gmail.com

Conflito de interesses: nada a declarar.

Recebido em: Mar. 02, 2019. Aprovado em: Mar. 02, 2020
Este é um artigo publicado em acesso aberto (Open Access) sob a licença Creative Commons distribuição e reprodução em qualquer meio, sem restrições desde que o trabalho original seja corretamente citado. 


\section{INTRODUÇÃO}

O hábito alimentar adquirido nos primeiros anos de vida repercute nas práticas alimentares, no crescimento e no desenvolvimento, além de influenciar o estado de saúde e nutrição ao longo da vida ${ }^{1,2}$. Nessa perspectiva, é fundamental a identificação de fatores associados ao início precoce e à inadequação da alimentação complementar com objetivo de estabelecer estratégias que possam viabilizar ações de saúde pública para a promoção e incorporação de práticas alimentares saudáveis ao longo da infância³.

Dados internacionais revelaram que, em 2017, apenas 40\% das crianças menores de 6 meses de idade receberam aleitamento materno exclusivo (AME) ${ }^{4}$. No Brasil, nas capitais brasileiras e no Distrito Federal, a mediana de AME foi de 1,8 mês, e a de aleitamento materno, de 11,2 meses, em 2008. É possível destacar ainda a introdução precoce de água, chás, sucos e comida de sal logo no primeiro mês de vida, bem como o consumo elevado de bolachas e/ou salgadinhos $(71,7 \%)$, refrigerantes $(11,6 \%)$ e café $(8,7 \%)$ entre crianças de $9-12$ meses $^{5}$. Dada a importância da amamentação para a saúde da criança e da mulher, que inclui proteção contra doenças infecciosas, agudas e crônicas, assim como para os estados emocional e psicológico, essa realidade é ainda preocupante ${ }^{6}$.

O abandono do aleitamento materno é determinado, principalmente, pela baixa escolaridade, menor renda, trabalho materno e fatores psicossociais, como sintomatologia depressiva ${ }^{7}$. Além disso, a ausência de uma rede de apoio social pode contribuir para a interrupção do aleitamento e a decisão por outras escolhas alimentares ${ }^{8}$. O suporte dos serviços de saúde às mães também é importante para a garantia da amamentação exclusiva e de sua continuidade em conjunto com outros alimentos ${ }^{6}$. Assim, considerando a multidimensionalidade dos cenários das práticas alimentares infantis, o papel da rede social e do apoio social da mulher deve merecer atenção por parte dos profissionais de saúde ${ }^{8}$. Em específico, a introdução de alimentos com elevado teor de açúcares, gorduras e proteínas na alimentação infantil antes do primeiro ano de vida tem sido relacionada com baixas escolaridade materna e renda familiar ${ }^{3}$.

Desde 1998, é recomendado que o consumo alimentar seja estudado com base nos alimentos, e não apenas em nutrientes isolados ${ }^{9}$. Assim, no Brasil, com o objetivo de identificar o padrão de alimentação e viabilizar a realização da Vigilância Alimentar e Nutricional (VAN) na rotina da atenção básica, o Ministério da Saúde recomenda a utilização do Formulário de Marcadores de Consumo Alimentar. Essa avaliação pelos profissionais das equipes de atenção básica com a obtenção de indicadores de forma regular constitui ainda uma ferramenta de cuidado e gestão das ações de alimentação e nutrição ${ }^{10}$. Entretanto, a baixa cobertura do Sistema de Vigilância Alimentar e Nutricional (SISVAN), sobretudo nas localidades menos desenvolvidas, mostra que a incorporação da VAN no cotidiano dos serviços de saúde não é priorizada, podendo comprometer a atenção nutricional ${ }^{11}$.

Pesquisas que contemplem a avaliação do consumo alimentar são essenciais para o estabelecimento das condições de saúde, pois possibilitam caracterizar as vulnerabilidades da população associadas à dieta ${ }^{12,13}$. Ainda, permitem propor medidas de intervenção direcionadas à garantia da saúde, particularmente importantes para crianças que têm na alimentação um dos principais determinantes do crescimento e desenvolvimento ${ }^{13}$.

Nessa perspectiva, o presente estudo tem como objetivo avaliar os marcadores do consumo alimentar de crianças menores de 5 anos de idade atendidas na Estratégia Saúde da Família (ESF) e verificar sua associação com o contexto social.

\section{MÉTODO}

Trata-se de um estudo que faz parte de uma pesquisa de avaliação multifacetada e multietápica da implantação das ações de alimentação e nutrição na ESF no estado da Paraíba. Entre os objetivos secundários do estudo, foi estabelecida a avaliação do consumo alimentar das crianças, com vistas a verificar a capacidade do Formulário de Marcadores de Consumo Alimentar no contexto da atenção básica ${ }^{10}$ de servir como ferramenta de cuidado e gestão das ações de alimentação e nutrição. 
O cenário da referida pesquisa, com coleta de dados relacionados às crianças menores de 5 anos de idade, foram os municípios do estado com população entre 30.000 e 149.999 habitantes, prioritários para ações voltadas à prevenção da obesidade infantil no contexto do Programa Saúde na Escola (PSE) ${ }^{14}$. Do total de 12 municípios, três foram excluídos, um por ser o único com cobertura parcial da ESF, outro por não possuir equipe de saúde do Programa Mais Médicos vinculada ao Núcleo Ampliado de Saúde da Família e o terceiro por sua inserção em outra proposta com características similares ao objeto da presente. Participaram do estudo 46 equipes de saúde da ESF apoiadas pelo Núcleo Ampliado de Saúde da Família e 17 creches vinculadas às equipes de saúde no contexto do PSE.

Foram consideradas como população de estudo as crianças de 0 a 59 meses de idade residentes nos municípios selecionados para participar da pesquisa, usuárias da ESF ( $N=$ 38.140). Por serem condições associadas a complicações no estado de saúde e nutrição infantil, crianças gêmeas, adotadas e de mães com idade inferior a 18 anos foram excluídas do estudo.

O cálculo do tamanho da amostra foi realizado considerando o nível de significância bicaudal de $5 \%(\alpha=0,05)$, intervalo de confiança de $95 \%$, poder estatístico de $90 \%(\beta=0,10)$, razão entre expostos e não expostos de 1:1, proporção esperada de ocorrência do desfecho no grupo de não expostos de $20 \%$ (prevalência de excesso de peso em crianças usada como indicador para decidir a inclusão de municípios como prioritários para o desenvolvimento de ações voltadas à prevenção da obesidade infantil ${ }^{14}$ ) e razão de prevalência esperada de 1,5 , indicando a necessidade de incluir no mínimo 790 indivíduos. A este número foram adicionados $15 \%$ para compensar possíveis perdas e controlar fatores de confusão, totalizando uma amostra de 909 crianças. O software Epi Info, versão 7.2, foi usado para realizar o cálculo.

Para cada município, a quantidade de equipes de saúde e de creches foi determinada proporcionalmente ao número de crianças menores de 5 anos residentes nele, contemplandose tanto equipes de saúde convencionais quanto do Programa Mais Médicos, respeitando a composição de cada município, e no mínimo uma creche por município. A quantidade de indivíduos também foi estabelecida de forma proporcional à população de crianças residentes em cada município. Não foi instituído um número a priori para cada equipe de saúde. Para as creches, o quantitativo de crianças foi definido de forma que permitisse completar o total previsto por município, considerando as observações realizadas no contexto das equipes de saúde.

A amostra foi selecionada em dois estágios. No primeiro, foram sorteadas aleatoriamente as equipes de saúde e creches, e no segundo estágio, as crianças. Para as equipes de saúde, as crianças foram, primeiramente, selecionadas de forma aleatória por ocasião do atendimento de puericultura no dia da coleta de dados. Em um segundo momento, foi realizada busca ativa das crianças nos domicílios, com base nos registros das equipes de saúde, sendo a seleção por sorteio aleatório. Nas creches, as crianças foram selecionadas probabilisticamente a partir da lista de disponibilidade delas nessas instituições, excluindo-se as que não estavam presentes no dia do trabalho de campo e as de maior idade na ocorrência de irmãos na mesma creche.

A coleta de dados foi realizada nas unidades de saúde e creches, no segundo semestre de 2018, por pesquisadores entrevistadores (profissionais e estudantes da área de saúde) com experiência prévia em trabalho de campo, o qual foi supervisionado por profissional capacitado. O controle de qualidade do estudo incluiu: treinamento e padronização dos entrevistadores, construção de manual de instruções e realização de estudo-piloto em um município do estado.

Foram obtidas informações relacionadas ao consumo alimentar e contexto social da criança, o qual contemplou dados sobre situação socioeconômica (classificação socioeconômica da família e trabalho da mãe fora de casa), apoio social (convivência da mãe com companheiro, funcionalidade familiar e suporte social), assistência social (frequência da criança à creche e benefício do Programa Bolsa Família) e segurança alimentar e nutricional da família.

As informações do consumo alimentar foram coletadas por meio do Formulário de Marcadores de Consumo Alimentar, considerando-se as orientações para a obtenção de marcadores de consumo alimentar na atenção básica ${ }^{10}$. Esse formulário é constituído de questões referidas ao dia anterior ao inquérito, incluindo comportamentos alimentares de risco para o desenvolvimento de obesidade infantil, voltadas para as crianças de acordo 
com a faixa etária: menores de 6 meses, de 6 a 23 meses e 29 dias e de 2 a 5 anos. Assim, os marcadores de consumo alimentar foram estabelecidos segundo a idade da criança: AME em crianças menores de 6 meses; aleitamento materno continuado, diversidade alimentar mínima e consumo de alimentos ultraprocessados em crianças de 6 a 23 meses e 29 dias; consumo de fruta, consumo de verduras e legumes, alimentos ultraprocessados e hábito de realizar no mínimo as três refeições principais do dia em crianças de 2 a 5 anos. Todos os cálculos foram realizados conforme as especificações do instrumento ${ }^{10}$.

A classificação socioeconômica da família baseou-se nos critérios da Associação Brasileira de Empresas de Pesquisa ${ }^{15}$, que são usados para estimar o poder de compra de famílias brasileiras. Para essa classificação, foram considerados o grau de instrução do chefe de família, o acesso a serviços públicos, a existência de vaso sanitário no domicílio, a contratação de empregada doméstica e a posse de bens. As famílias foram classificadas nos níveis socioeconômicos $A / B / C$ (pontuação de 17 a 100) e D/E (pontuação de 0 a 16).

A funcionalidade familiar foi medida por meio do APGAR Familiar, um questionário composto por cinco questões, uma para cada domínio de avaliação: adaptation (adaptação), que compreende os recursos familiares oferecidos quando se faz a necessária assistência; partnership (companheirismo), que se refere à reciprocidade nas comunicações familiares e na solução de problemas; growth (desenvolvimento), que é relativo à disponibilidade da família para mudanças de papéis e desenvolvimento emocional; affection (afetividade), que compreende a intimidade e as interações emocionais no contexto familiar; e resolve (capacidade resolutiva), que está associada à decisão, determinação ou resolutividade em uma unidade familiar. $O$ instrumento apresenta três opções de respostas - sempre, que equivale a 2 pontos; algumas vezes, 1 ponto; e nunca, 0 ponto -, com pontuação total que varia de 0 a 10 . As famílias com pontuação de 0 a 3 são classificadas com elevada disfunção familiar; de 4 a 6, moderada disfunção familiar; e de 7 a 10, boa funcionalidade familiar ${ }^{16,17}$. Para fins de análise, no presente estudo as famílias foram classificadas como funcional ou disfuncional (elevada e moderada disfunção familiar).

Para o suporte social, foi utilizado o questionário do Medical Outcomes Study, que é composto por 19 itens em cinco dimensões de apoio social: material (quatro perguntas), afetivo (três perguntas), emocional (quatro perguntas), informação (quatro perguntas) e interação social (quatro perguntas), recomendando-se a agregação das dimensões emocional e informação. Para todas as perguntas, as opções de respostas são cinco: sempre, que equivale a 5 pontos; quase sempre, 4 pontos; às vezes, 3 pontos; raramente, 2 pontos; e nunca, 1 ponto. Em cada uma das dimensões, os escores dos itens foram adicionados. O escore total foi dividido pelo escore máximo possível para a dimensão e multiplicado por $100^{18,19}$. Os escores foram dicotomizados com base na mediana mais frequentemente encontrada nas análises das diferentes dimensões de apoio social. Desta forma, foi utilizado como ponto de corte único o "escore > 90" como indicativo do mais alto nível de apoio social.

Para avaliar a situação de segurança alimentar e nutricional, foi aplicada a Escala Brasileira de Insegurança Alimentar com 14 itens ${ }^{20}$. Essa é uma escala validada que possibilita classificar as famílias em diferentes graus de insegurança alimentar: segurança alimentar, quando todas as questões são respondidas de forma negativa; insegurança alimentar leve, quando há até 5 questões com respostas positivas; insegurança alimentar moderada, nos casos de 6 a 9 respostas positivas; e insegurança alimentar grave, para 10 a 14 respostas positivas.

O grupo de variáveis independentes foi formado por: trabalho materno fora de casa, classificação socioeconômica da família (A/B/C e D/E), convivência da mãe com companheiro, funcionalidade familiar (funcional e disfuncional), apoio social (alto e baixo), frequência da criança à creche, benefício do Programa Bolsa Família (PBF) e segurança alimentar e nutricional da família (segurança alimentar/insegurança alimentar leve e insegurança alimentar moderada/ grave). Os marcadores de consumo alimentar das crianças, segundo a faixa etária, foram os desfechos de interesse: AME (sim e não) para crianças menores de 6 meses; aleitamento materno continuado (sim e não), diversidade alimentar (sim e não) e consumo de alimentos ultraprocessados (sim e não) para crianças de 6-23 meses e 29 dias; e consumo de frutas (sim 
e não), consumo de verduras (sim e não), consumo de alimentos ultraprocessados (sim e não) e realização das três refeições principais do dia (sim e não) para crianças de 2-5 anos.

Os dados coletados foram organizados em planilhas eletrônicas e digitados em dupla entrada em um banco de dados customizado com verificações de consistência e restrições de intervalo. Foi utilizado para análises estatísticas o banco produzido após verificação de análise de consistência dos dados digitados.

Foi empregado o teste estatístico qui-quadrado, considerando o nível de significância a igual a 5\%. As análises foram realizadas com o software Stata, versão 12.0.

A pesquisa foi aprovada pelo Comitê de Ética em Pesquisa da Universidade Estadual da Paraíba (CAAE 71609317.9.0000.5187). Todos os participantes da pesquisa assinaram o Termo de Consentimento Livre e Esclarecido como condição necessária à participação no estudo e receberam uma via dele. Este estudo não apresenta conflitos de interesse por nenhum de seus autores.

\section{RESULTADOS}

Participaram do estudo 897 crianças, sendo que 893 tiveram os dados completos de consumo alimentar, das quais 20,5\% eram menores de 6 meses e 31,4\% tinham de 6 a 23 meses e 29 dias. Em relação ao perfil materno infantil, as mães, em sua maioria, informaram não trabalhar fora de casa $(71,3 \%)$, foram classificadas no nível socioeconômico D/E (64,3\%), conviviam com o companheiro $(74,8 \%)$, viviam em famílias funcionais $(70,2 \%)$ e percebiam-se com baixo apoio social (54,8\%). Do total de crianças, $57,2 \%$ não frequentavam creches, $67,4 \%$ eram beneficiárias do PBF e 75,7\% estavam em segurança alimentar/insegurança alimentar leve (Tabela 1).

Em relação ao consumo alimentar do dia anterior à coleta de dados, foi observado que $40,4 \%$ das crianças menores de 6 meses estavam em AME. A não convivência da mãe com companheiro se associou ao aleitamento materno não exclusivo $(p=0,041)$ (Tabela 2).

Quanto às práticas alimentares, foi constatado que $56,8 \%$ das crianças de 6 a 23 meses e 29 dias estavam em aleitamento materno continuado. Foram fatores associados à interrupção do aleitamento materno: trabalho materno fora de casa $(p=0,003)$, nível socioeconômico $A / B / C(p=0,034)$, não convivência da mãe com companheiro $(p=0,042)$ e frequentar creche $(p=0,008)$. Em relação aos indicadores de qualidade da dieta, foi verificado que apenas $18,2 \%$ das crianças tinham diversidade alimentar mínima, associando-se ao benefício do PBF $(p=0,033)$ e à insegurança alimentar moderada/grave $(p=0,039)$. Foi percebido ainda que $51,1 \%$ das crianças consumiram alimentos ultraprocessados no dia anterior à entrevista, com pior situação naquelas não beneficiárias do $\operatorname{PBF}(p=0,043)$ (Tabela 3).

Nas crianças de 2 a 5 anos de idade, $74,9 \%$ e $61,2 \%$ consumiram fruta e verduras e legumes no dia anterior, respectivamente. $O$ baixo consumo de fruta associou-se à ausência de trabalho materno fora de casa $(p=0,023)$, contexto familiar disfuncional $(p=0,005)$, baixo apoio social $(p=0,032)$ e insegurança alimentar moderada/grave $(p=0,001)$. O consumo de alimentos ultraprocessados foi de $25,8 \%$, sendo mais frequente entre as crianças que não frequentavam creche $(p=0,019)$ (Tabela 4).

Quanto ao hábito de realizar no mínimo as três refeições principais do dia, 92,4\% das crianças as realizavam. Estiveram estatisticamente associadas à ausência desse hábito a não frequência da criança à creche $(p=0,019)$ e o não benefício do $\operatorname{PBF}(p=0,034)$ (Tabela 5$)$.

\section{DISCUSSÃO}

Para a criança ter uma alimentação saudável, é recomendado AME até os 6 meses e complementado até os 2 anos ou mais ${ }^{21}$. Neste estudo, a prevalência de AME foi de 40,4\%, similar às reportadas nacionalmente de $38,6 \%{ }^{22}$ e $41 \%{ }^{5}$, bem como a encontrada de $41,1 \%$ em outra pesquisa que utilizou o mesmo instrumento ${ }^{23}$. Essas taxas se aproximam da prevalência de amamentação exclusiva de $37 \%$ estimada para crianças de países de baixa e média renda ${ }^{24}$. Entretanto, são superiores às registradas no país mais recentemente $(20,6 \%)^{6}$ e em metanálise de estudos epidemiológicos brasileiros $(25 \%)^{25}$. Em relação ao aleitamento materno continuado, a prevalência descrita neste estudo (56,8\%) é similar à encontrada no âmbito nacional $(56 \%)^{6}$, 
Tabela 1. Descrição das características do contexto social da amostra de crianças menores de 5 anos de idade usuárias da Estratégia Saúde da Família, residentes em municípios do estado da Paraíba/PB, Brasil, 2018 (N=893)

\begin{tabular}{|c|c|c|c|c|c|c|c|c|}
\hline \multirow{3}{*}{ Variáveis } & \multicolumn{8}{|c|}{ Idade da criança } \\
\hline & \multicolumn{2}{|c|}{$\begin{array}{c}\text { 0-5 meses e } 29 \\
\text { dias }\end{array}$} & \multicolumn{2}{|c|}{ 6-23 meses e 29 dias } & \multicolumn{2}{|c|}{ 2-5 anos } & \multicolumn{2}{|c|}{ Total da amostra } \\
\hline & $\mathbf{n}$ & $\%$ & $\mathbf{n}$ & $\%$ & $\mathbf{n}$ & $\%$ & $\mathbf{n}$ & $\%$ \\
\hline \multicolumn{9}{|l|}{ Trabalho materno fora de casa } \\
\hline Sim & 52 & 28,4 & 54 & 19,3 & 150 & 34,9 & 256 & 28,7 \\
\hline Não & 131 & 71,6 & 226 & 80,7 & 280 & 65,1 & 637 & 71,3 \\
\hline \multicolumn{9}{|c|}{ Classificação socioeconômica da família } \\
\hline$A / B / C$ & 84 & 45,9 & 95 & 33,9 & 140 & 32,6 & 319 & 35,7 \\
\hline $\mathrm{D} / \mathrm{E}$ & 99 & 54,1 & 185 & 66,1 & 290 & 67,4 & 574 & 64,3 \\
\hline \multicolumn{9}{|c|}{ Convivência da mãe com o companheiro } \\
\hline Sim & 157 & 85,8 & 219 & 78,2 & 292 & 67,9 & 668 & 74,8 \\
\hline Não & 26 & 14,2 & 61 & 21,8 & 138 & 32,1 & 225 & 25,2 \\
\hline \multicolumn{9}{|l|}{ Funcionalidade familiar } \\
\hline Família funcional & 154 & 84,2 & 190 & 67,9 & 283 & 65,8 & 627 & 70,2 \\
\hline Família disfuncional & 29 & 15,8 & 90 & 32,1 & 147 & 34,2 & 266 & 29,8 \\
\hline \multicolumn{9}{|l|}{ Apoio social } \\
\hline Alto & 108 & 59,0 & 123 & 43,9 & 173 & 40,2 & 404 & 45,2 \\
\hline Baixo & 75 & 41,0 & 157 & 56,1 & 257 & 59,8 & 489 & 54,8 \\
\hline \multicolumn{9}{|l|}{ Frequência da criança à creche } \\
\hline Sim & 14 & 7,6 & 48 & 17,14 & 320 & 74,4 & 382 & 42,8 \\
\hline Não & 169 & 92,4 & 232 & 82,9 & 110 & 25,6 & 511 & 57,2 \\
\hline \multicolumn{9}{|c|}{ Benefício do Programa Bolsa Família } \\
\hline Sim & 86 & 47,0 & 179 & 63,9 & 337 & 78,4 & 602 & 67,4 \\
\hline Não & 97 & 53,0 & 101 & 36,1 & 93 & 21,6 & 291 & 32,6 \\
\hline \multicolumn{9}{|c|}{ Segurança alimentar e nutricional da família } \\
\hline $\begin{array}{l}\text { Segurança alimentar/ } \\
\text { insegurança alimentar leve }\end{array}$ & 144 & 78,7 & 200 & 71,4 & 332 & 77,2 & 676 & 75,7 \\
\hline $\begin{array}{l}\text { Insegurança alimentar } \\
\text { moderada/grave }\end{array}$ & 39 & 21,3 & 80 & 28,6 & 98 & 22,8 & 217 & 24,3 \\
\hline
\end{tabular}

um pouco superior à obtida em pesquisa também baseada nos marcadores de consumo da atenção básica $(44,4 \%)$ e um pouco inferior à estimada nos países de baixa e média renda $(63 \%)^{24}$. Tendo em vista a meta de aumentar o AME em pelo menos $50 \%$ até $2025^{26}$, esses achados devem ser motivo de preocupação. Nesse sentido, a Estratégia Amamenta e Alimenta Brasil deve ser priorizada, considerando que unidades básicas de saúde certificadas por ela têm mostrado efeitos positivos no AME27.

Entre as crianças de 6 a 23 meses e 29 dias, é possível presumir uma alimentação monótona, pois somente $18,2 \%$ delas consumiram seis grupos de alimentos no dia anterior à entrevista, o que pode contribuir para o agravamento das principais deficiências nutricionais ${ }^{28}$. Estudos prévios também mostraram que a qualidade da dieta das crianças brasileiras se caracteriza pela 
Tabela 2. Aleitamento materno exclusivo de crianças menores de 6 meses de idade usuárias da Estratégia Saúde da Família, residentes em municípios do estado da Paraíba/PB, Brasil, 2018

\begin{tabular}{|c|c|c|c|c|c|}
\hline \multirow{3}{*}{ Variáveis } & \multicolumn{4}{|c|}{ Aleitamento materno exclusivo } & \multirow{3}{*}{ p-valor } \\
\hline & \multicolumn{2}{|c|}{ Sim } & \multicolumn{2}{|c|}{ Não } & \\
\hline & $\mathbf{n}$ & $\%$ & $\mathbf{n}$ & $\%$ & \\
\hline \multicolumn{5}{|l|}{ Trabalho materno fora de casa } & \multirow{3}{*}{0,179} \\
\hline Sim & 17 & 32,7 & 35 & 67,3 & \\
\hline Não & 57 & 43,5 & 74 & 56,5 & \\
\hline \multicolumn{5}{|l|}{ Classificação socioeconômica da família } & \multirow{3}{*}{0,359} \\
\hline $\mathrm{A} / \mathrm{B} / \mathrm{C}$ & 37 & 44,1 & 47 & 55,9 & \\
\hline$D / E$ & 37 & 37,4 & 62 & 62,6 & \\
\hline \multicolumn{5}{|l|}{ Convivência da mãe com o companheiro } & \multirow{3}{*}{0,041} \\
\hline Sim & 68 & 43,3 & 89 & 56,7 & \\
\hline Não & 6 & 23,1 & 20 & 76,9 & \\
\hline \multicolumn{5}{|l|}{ Funcionalidade familiar } & \multirow{3}{*}{0,476} \\
\hline Família funcional & 64 & 41,6 & 90 & 58,4 & \\
\hline Família disfuncional & 10 & 34,5 & 19 & 65,5 & \\
\hline \multicolumn{5}{|l|}{ Apoio social } & \multirow{3}{*}{0,684} \\
\hline Alto & 45 & 41,7 & 63 & 58,3 & \\
\hline Baixo & 29 & 38,7 & 46 & 61,3 & \\
\hline \multicolumn{5}{|l|}{ Frequência da criança à creche } & \multirow{3}{*}{0,848} \\
\hline Sim & 6 & 42,9 & 8 & 57,1 & \\
\hline Não & 68 & 40,2 & 101 & 59,8 & \\
\hline \multicolumn{5}{|l|}{ Benefício do Programa Bolsa Família } & \multirow{3}{*}{0,254} \\
\hline Sim & 31 & 36,1 & 55 & 63,9 & \\
\hline Não & 43 & 44,3 & 54 & 55,7 & \\
\hline \multicolumn{5}{|l|}{ Segurança alimentar e nutricional da família } & \multirow{3}{*}{0,515} \\
\hline $\begin{array}{l}\text { Segurança alimentar/insegurança alimentar } \\
\text { leve }\end{array}$ & 60 & 41,7 & 84 & 58,3 & \\
\hline Insegurança alimentar moderada/grave & 14 & 35,9 & 25 & 64,1 & \\
\hline
\end{tabular}

falta de diversidade ${ }^{1,28}$. Esse parece não ser apenas um problema no Brasil, revelando-se, nos Estados Unidos, por exemplo, baixa ingestão de todos os grupos alimentares entre menores de 11 meses de idade ${ }^{29}$.

Para as crianças de 2 a 5 anos de idade, as prevalências do consumo de fruta $(74,9 \%)$ e verduras e legumes $(61,2 \%)$ condizem com resultados nacionais que ressaltaram maior consumo de fruta $(89,6 \%)$ em relação à ingestão de verduras de folhas $(50,4 \%)^{30}$. Considerando que há poucos estudos acerca da ingestão de frutas e verduras entre as crianças brasileiras, contudo com evidências do consumo deficitário $(<50 \%)$ em todos os grupos etários e regiões do Brasi $^{31}$, a suscetibilidade relacionada ao aporte recomendado de micronutrientes deve causar suspeitas ${ }^{30}$.

Hábitos alimentares não saudáveis na infância, caracterizados pelo consumo de alimentos ultraprocessados, têm impacto negativo na saúde das crianças, como o aumento 


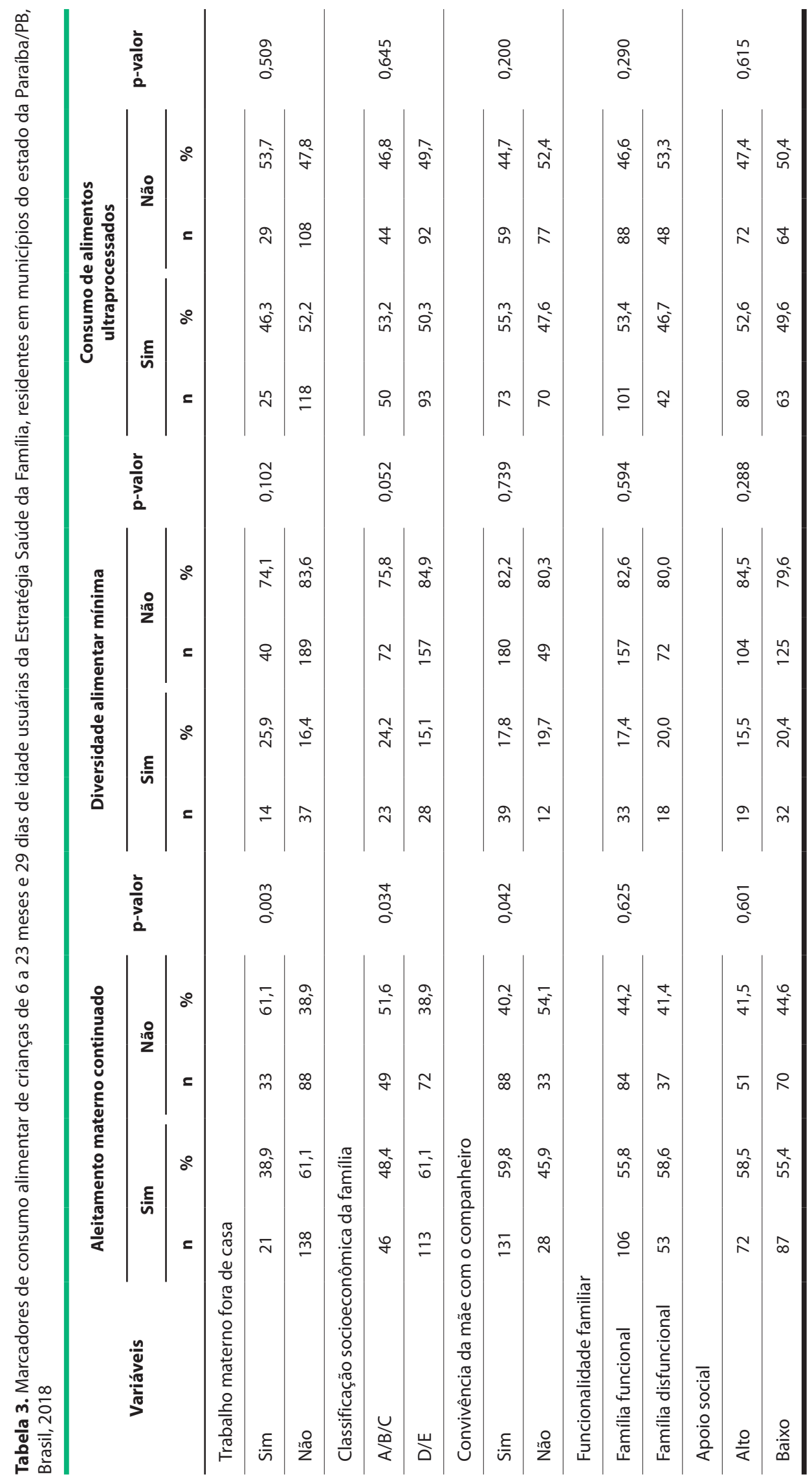




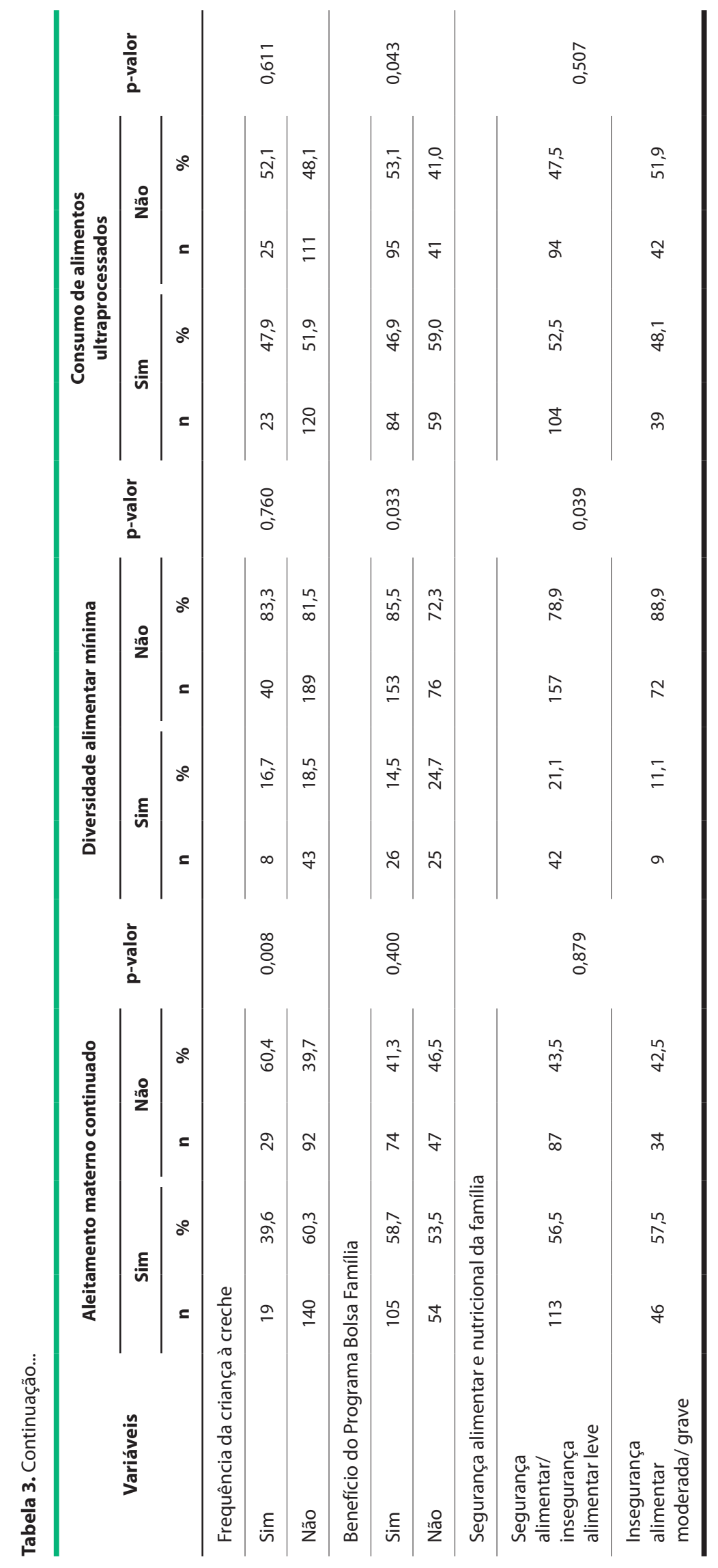




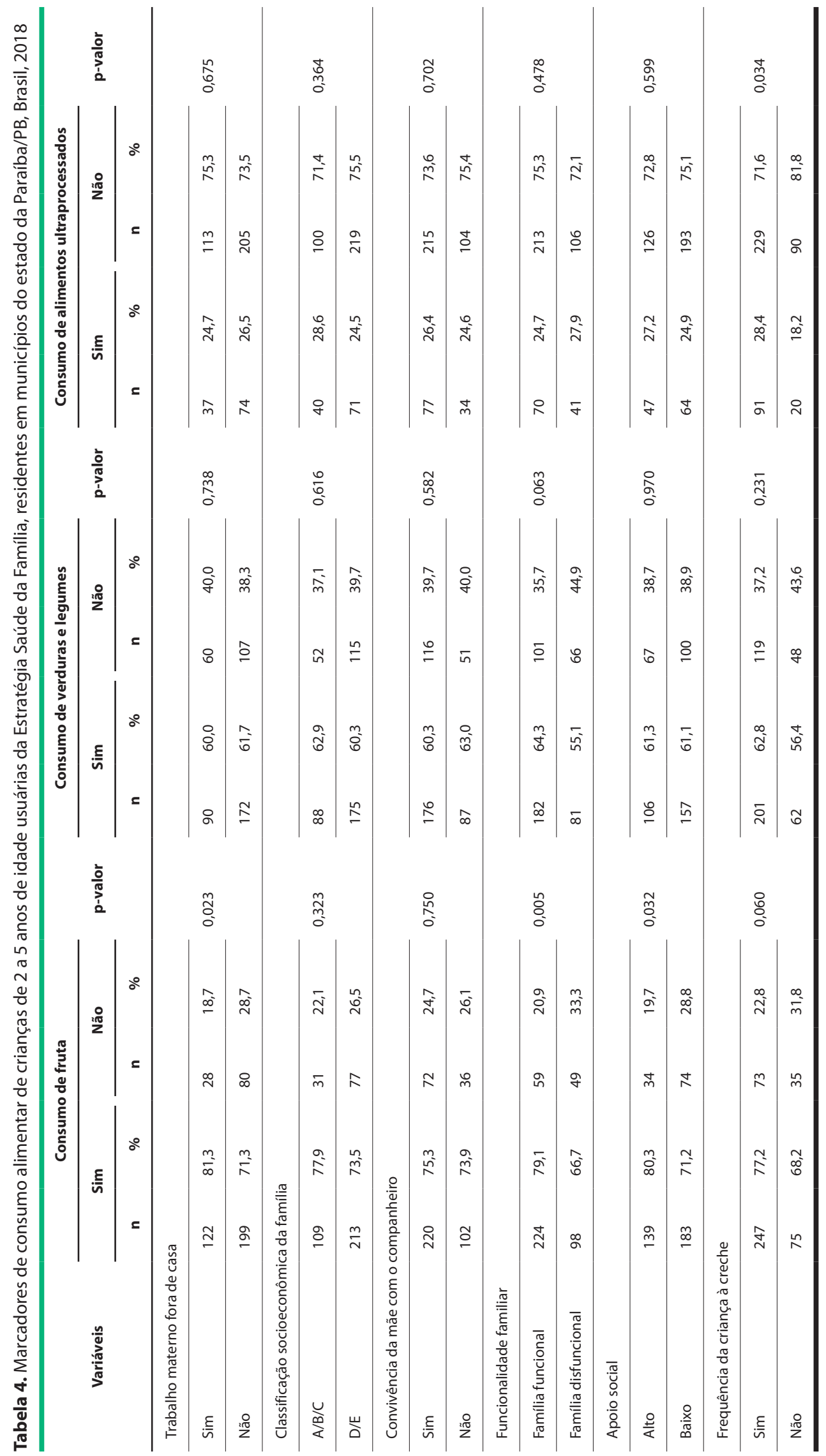




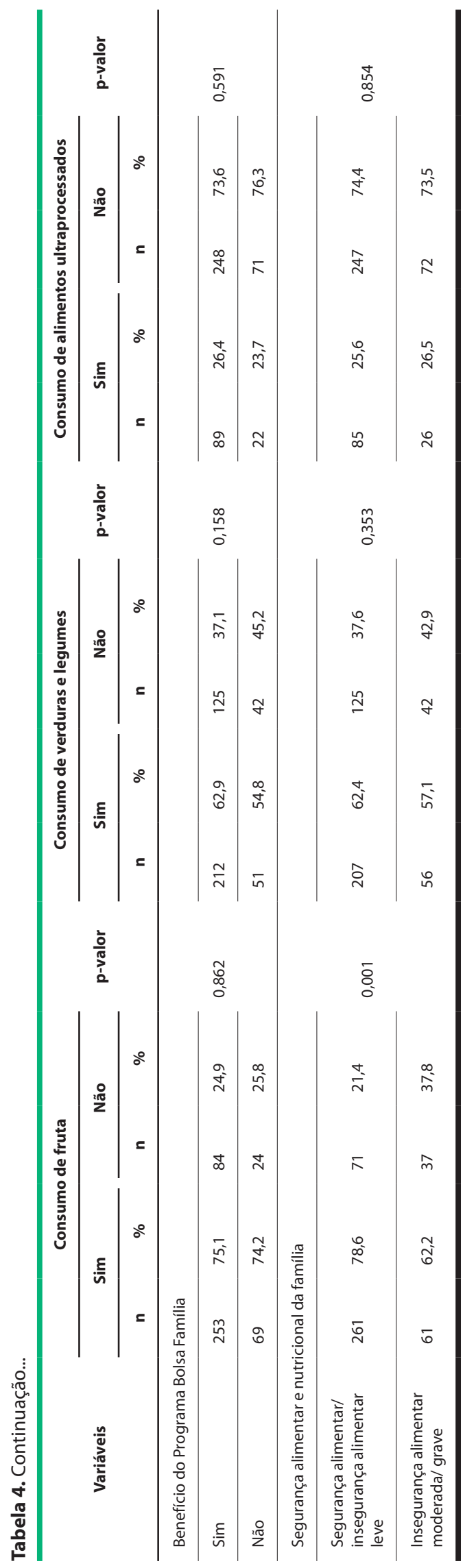


Tabela 5. Hábito de realizar no mínimo as três refeições principais do dia de crianças de 2 a 5 anos de idade usuárias da Estratégia Saúde da Família, residentes em municípios do estado da Paraíba/PB, Brasil, 2018

\begin{tabular}{|c|c|c|c|c|c|}
\hline \multirow{3}{*}{ Variáveis } & \multicolumn{4}{|c|}{$\begin{array}{l}\text { Hábitos de realizar as três refeições principais do } \\
\text { dia }\end{array}$} & \multirow{3}{*}{ p-valo } \\
\hline & \multicolumn{2}{|c|}{ Sim } & \multicolumn{2}{|c|}{ Não } & \\
\hline & $\mathbf{n}$ & $\%$ & $\mathbf{n}$ & $\%$ & \\
\hline \multicolumn{5}{|l|}{ Trabalho materno fora de casa } & \multirow{3}{*}{0,579} \\
\hline Sim & 140 & 93,3 & 10 & 6,7 & \\
\hline Não & 259 & 91,8 & 23 & 80,2 & \\
\hline \multicolumn{5}{|l|}{ Classificação socioeconômica da família } & \multirow{3}{*}{0,650} \\
\hline $\mathrm{A} / \mathrm{B} / \mathrm{C}$ & 130 & 91,6 & 12 & 8,4 & \\
\hline $\mathrm{D} / \mathrm{E}$ & 270 & 92,8 & 21 & 7,2 & \\
\hline \multicolumn{5}{|l|}{ Convivência da mãe com o companheiro } & \multirow{3}{*}{0,851} \\
\hline Sim & 273 & 92,5 & 22 & 7,5 & \\
\hline Não & 127 & 92,0 & 11 & 8,0 & \\
\hline \multicolumn{5}{|l|}{ Funcionalidade familiar } & \multirow{3}{*}{0,313} \\
\hline Família funcional & 265 & 93,3 & 19 & 6,7 & \\
\hline Família disfuncional & 135 & 90,6 & 14 & 9,4 & \\
\hline \multicolumn{5}{|l|}{ Apoio social } & \multirow{3}{*}{0,661} \\
\hline Alto & 161 & 93,1 & 12 & 6,9 & \\
\hline Baixo & 239 & 91,9 & 21 & 8,1 & \\
\hline \multicolumn{5}{|l|}{ Frequência da criança à creche } & \multirow{3}{*}{0,019} \\
\hline $\operatorname{Sim}$ & 304 & 94,1 & 19 & 5,9 & \\
\hline Não & 96 & 87,3 & 14 & 12,7 & \\
\hline \multicolumn{5}{|l|}{ Benefício do Programa Bolsa Família } & \multirow{3}{*}{0,034} \\
\hline Sim & 318 & 93,8 & 21 & 6,2 & \\
\hline Não & 82 & 87,2 & 12 & 12,8 & \\
\hline \multicolumn{5}{|l|}{ Segurança alimentar e nutricional da família } & \multirow{3}{*}{0,839} \\
\hline $\begin{array}{l}\text { Segurança alimentar/ insegurança alimentar } \\
\text { leve }\end{array}$ & 309 & 92,2 & 26 & 7,8 & \\
\hline Insegurança alimentar moderada/grave & 91 & 92,9 & 7 & 7,1 & \\
\hline
\end{tabular}

das concentrações de colesterol total, das low-density lipoprotein e do índice de massa corporal, ainda nos primeiros anos de vida ${ }^{3}$. No Brasil, uma a cada três crianças menores de 2 anos consumiu refrigerante e $60,8 \%$ comeram biscoitos ou bolos, segundo dados recentes $\mathrm{s}^{32}$. A alta prevalência no consumo desses alimentos também foi observada em outras pesquisas ${ }^{23,33}$, confirmando-se neste estudo. Apesar de que, nas crianças de 2 a 5 anos, esse marcador foi menos expressivo, mais de um quarto delas consumia alimentos do grupo, proporção que variou de $13,5 \%$ a $65,4 \%$ entre indivíduos da mesma faixa etária em estudo nacional ${ }^{30}$. Acredita-se que o marketing agressivo torna esse grupo de alimentos atraentes e desejados, principalmente pelo público infantil. Diante desse cenário, cabe aos profissionais de saúde, principalmente ao nutricionista, o desenvolvimento de ações de incentivo à alimentação saudável, realizadas em momento oportuno ${ }^{33}$. 
Neste estudo, além de se verificarem altas prevalências de inadequações no consumo alimentar das crianças, foi possível constatar associações com as características do contexto social. No que se refere ao tempo de aleitamento materno, a importância da figura do companheiro, tanto na descontinuidade do componente exclusivo ${ }^{7}$ quanto do complementar ${ }^{34}$, foi reafirmada por meio deste estudo. Essa relação tem por base a importância do suporte do companheiro, outros familiares e membros da rede social, seja na realização de tarefas domésticas, no auxílio financeiro ou no apoio ao cuidado com a criança ${ }^{35}$.

A interrupção total do aleitamento materno nas crianças deste trabalho esteve ainda associada ao trabalho materno fora do lar, o que pode estar relacionado ao afastamento físico entre a mãe e a criança por causa das responsabilidades laborais ${ }^{36}$, conforme constatado em outro estudo ${ }^{34}$. Dessa forma, é necessário garantir às mulheres, além de licença-maternidade, locais de trabalho e centros de cuidados infantis adequados que favoreçam a manutenção da amamentação ${ }^{34,36}$, o que parece não ser a realidade da população observada, pois frequentar a creche também representou uma exposição importante nesse desfecho. As creches devem possuir estrutura adequada ao acolhimento de crianças em aleitamento materno, com equipamentos para a estocagem de leite materno e funcionários treinados, por exemplo, conforme sugestões de pesquisadores que obtiveram resultados similares aos aqui apresentados ${ }^{37}$. Deve ser destacado, adicionalmente, o papel essencial dos gestores, que precisam adotar atitude proativa de estímulo e apoio ao aleitamento materno na instituição escolar ${ }^{21}$.

Por fim, a melhor situação socioeconômica, como exposição associada à descontinuação do aleitamento materno observada no atual estudo, reforça achados anteriores com base em dados nacionais ${ }^{6}$ e de países de baixa e média renda ${ }^{24}$. Ainda, converge com os resultados de uma revisão da literatura mundial com foco nos fatores associados à manutenção da amamentação por 12 meses ou mais, a qual argumenta o benefício econômico da amamentação como possível precursor de comportamento positivo nas camadas mais pobres ${ }^{36}$.

Nas crianças de 6 a 23 meses e 29 dias, a maior frequência de não diversidade alimentar mínima entre as beneficiárias do PBF autentica a visão de uma revisão da literatura que aponta esse programa como promotor do acesso aos alimentos, contudo não necessariamente acompanhado por aumento da qualidade nutricional da alimentação ${ }^{38}$. Nesse sentido, o desenvolvimento de ações que promovam a educação alimentar e nutricional nesse grupo é considerado fundamental para a adequação do $\mathrm{PBF}^{39}$. Ainda, em razão de suas condições sociais, é possível que essas crianças residam em domicílios em pior situação de segurança alimentar, com prejuízos à diversidade e qualidade da dieta ${ }^{28}$, o que também corrobora os achados do presente estudo para essa condição.

A insegurança alimentar moderada/grave das famílias também foi um fator importante no baixo consumo de fruta nas crianças de 2 a 5 anos de idade, similar à tendência decrescente no consumo de frutas/sucos naturais, à medida que a insegurança alimentar aumentava, observada anteriormente ${ }^{40}$. A insegurança alimentar associa-se à qualidade da alimentação, com redução na ingestão de frutas e hortaliças e aumento no consumo de carboidratos refinados e gorduras ${ }^{41}$. Apesar de que outras condições também associadas ao consumo de frutas nas crianças do atual estudo têm sido pouco estudadas, é possível sustentar que esse resultado possa estar relacionado à falta de oportunidades de trabalho materno, que pode comprometer o acesso à alimentação saudável ${ }^{42}$, bem como à influência das relações interfamiliares e do ambiente doméstico nas preferências e no padrão de alimentação ${ }^{43}$.

Resultados prévios, que apontaram diferenças entre os padrões alimentares dentro e fora da creche, com melhor qualidade nutricional em um padrão "tradicional" dentro da creche ${ }^{44}$, e em relação ao benefício do PBF, sendo menor o consumo de alimentos processados e ultraprocessados entre beneficiários ${ }^{39}$, são reforçados por meio do atual estudo. Dessa forma, deve ser ressaltada a importância das creches e do PBF no padrão alimentar das crianças ${ }^{39,44}$.

O hábito de não realizar no mínimo as três refeições principais do dia, apesar de ter sido um marcador verificado em poucas crianças do atual estudo, foi mais frequente em crianças não assistidas em creches e entre as não beneficiárias do PBF. Essas relações podem decorrer da importância das creches em crianças socialmente vulneráveis como forma de suprir a 
carência alimentar no contexto domiciliar ${ }^{45}$ e do PBF na utilização da renda familiar para a compra de alimentos ${ }^{46}$.

Como limitações deste estudo, devem ser destacadas aquelas inerentes a pesquisas realizadas com informações autorreferidas, sujeitas a vieses de recordatório ou influenciadas pelo fato de os entrevistados conhecerem os comportamentos socialmente aceitos ${ }^{47}$. Apesar das suas desvantagens nas estimativas do consumo alimentar, a opção pelo uso do Formulário de Marcadores de Consumo Alimentar deve ser defendida por ser um método prático e informativo que permite simplificar a obtenção e a análise dos dados. Além disso, seu tamanho reduzido pode diminuir a sobrecarga no entrevistado e a omissão de dados importantes, como pode acontecer nos questionários de frequência alimentar ${ }^{48}$. Nesse sentido, foi possível mostrar que sua utilização pode ser uma importante ferramenta para a equipe de saúde na identificação de crianças vulneráveis ao consumo alimentar inadequado, no planejamento e organização das ações de cuidado nutricional local, no aprimoramento da qualidade dos serviços e na melhoria da resolubilidade, atuando de forma efetiva sobre os determinantes dos problemas alimentares que acometem a população de sua área.

Com este estudo, puderam ser identificados marcadores de consumo alimentar inadequados em crianças menores de 5 anos, incluindo a amamentação exclusiva e complementar, a diversidade alimentar mínima e a qualidade da alimentação. Além disso, foi possível verificar a associação dos marcadores com o contexto social da criança, sendo importantes as oportunidades de trabalho materno, o apoio social e do companheiro, os benefícios sociais (institucionalização em creches e benefício do PBF) e o acesso à alimentação. Apesar das limitações do instrumento utilizado, é evidente sua capacidade de identificar riscos associados ao consumo alimentar em crianças e de apoiar as políticas públicas de saúde a partir do SISVAN.

\section{REFERÊNCIAS}

1. Carvalho CA, Fonsêca PCA, Nobre LN, Silva MA, Pessoa MC, Ribeiro AQ, et al. Fatores associados aos padrões alimentares no segundo semestre de vida. Cien Saude Colet. 2020;25(2):449-59. http://dx.doi. org/10.1590/1413-81232020252.12982018. PMid:32022186.

2. Karnopp EVN, Vaz JS, Schafer AA, Muniz LC, Souza RLV, Santos I, et al. Consumo alimentar de crianças menores de seis anos conforme o grau de processamento. J Pediatr. 2017;93(1):70-8. http://dx.doi. org/10.1016/j.jped.2016.04.007. PMid:27393684.

3. Dallazen C, Silva SA, Gonçalves VSS, Nilson EAF, Crispim SP, Lang RMF, et al. Introdução de alimentos não recomendados no primeiro ano de vida e fatores associados em crianças de baixo nível socioeconómico. Cad Saude Publica. 2018;34(2):e00202816. http://dx.doi.org/10.1590/0102-311x00202816. PMid:29489953.

4. World Health Organization. United Nations Children's Fund. Tracking progress for breastfeeding policies and programmes: Global breastfeeding scorecard 2017. Geneva: WHO; 2017.

5. Brasil. Ministério da Saúde. II Pesquisa de Prevalência de Aleitamento Materno nas Capitais Brasileiras e Distrito Federal [Internet]. Brasília: Editora MS; 2009 [citado em 2019 fev 12]. Disponível em: http://bvsms. saude.gov.br/bvs/publicacoes/pesquisa_prevalencia_aleitamento_materno.pdf

6. Flores TR, Nunes BP, Neves RG, Wendt AT, Costa CS, Wehrmeister FC, et al. Consumo de leite materno e fatores associados em crianças menores de dois anos: Pesquisa Nacional de Saúde, 2013. Cad Saude Publica. 2017;33(11):e00068816. http://dx.doi.org/10.1590/0102-311x00068816. PMid:29166476.

7. Machado MCM, Assis KF, Oliveira FCC, Ribeiro AQ, Araújo RMA, Cury AF, et al. Determinantes do abandono do aleitamento materno exclusivo: fatores psicossociais. Rev Saude Publica. 2014;48(6):985-94. http:// dx.doi.org/10.1590/S0034-8910.2014048005340. PMid:26039402.

8. Morgado $\mathrm{CMC}$, Werneck GL, Hasselmann MH. Rede de apoio social e práticas alimentares de crianças no quarto mês de vida. Cien Saude Colet. 2013;18(2):367-76. http://dx.doi.org/10.1590/S141381232013000200008.

9. World Health Organization. Preparation and use of food basead dietary guidelines. Geneva: WHO; 1998.

10. Brasil. Ministério da Saúde. Orientações para avaliação de marcadores de consumo alimentar na atenção básica [Internet]. Brasília: Editora MS; 2015 [citado em 2019 fev 12]. Disponível em: http://bvsms.saude. gov.br/bvs/publicacoes/marcadores_consumo_alimentar_atencao_basica.pdf 
11. Nascimento FA, Silva AS, Jaime PC. Cobertura da avaliação do estado nutricional no Sistema de Vigilância Alimentar e Nutricional brasileiro: 2008 a 2013. Cad Saude Publica. 2017;33(12):e00161516. http://dx.doi. org/10.1590/0102-311x00161516. PMid:29267692.

12. Pedraza DF, Menezes TN. Questionários de Frequência de Consumo Alimentar desenvolvidos e validados para população do Brasil: revisão da literatura. Cien Saude Colet. 2015;20(9):2697-720. http://dx.doi. org/10.1590/1413-81232015209.12602014. PMid:26331503.

13. Sotero AM, Cabral PM, Silva GAP. Fatores socioeconômicos, culturais e demográficos maternos associados ao padrão alimentar de lactentes. Rev Paul Pediatr. 2015;33(4):445-52. http://dx.doi.org/10.1016/j. rpped.2015.03.006. PMid:26298652.

14. Brasil. Portaria $n^{\circ} 2.706$, de 18 de outubro de 2017. Lista os municípios que finalizaram a adesão ao Programa Saúde na Escola para o ciclo 2017/2018 e os habilita ao recebimento do teto de recursos financeiros pactuados em Termo de Compromisso e repassa recursos financeiros para Municípios prioritários para ações de prevenção da obesidade infantil com escolares. Diário Oficial da União, Brasília, 20 de outubro de 2017; Seção 1. p. 49.

15. Associação Brasileira de Empresas de Pesquisa. Critério de classificação econômica Brasil. São Paulo: ABEP; 2016.

16. Smilkstein G. The family APGAR: a proposal for a family function test and its use by physicians. J Fam Pract 1978;6(6):1231-9. PMid:660126.

17. Good MV, Smilkstein G, Good BJ, Shaffer T, Arons T. The family APGAR index: a study of construct validity. J Fam Pract. 1979;8(3):577-82.

18. Griep RH, Chor D, Faerstein E, Werneck GL, Lopes CS. Validade de constructo de escala de apoio social do Medical Outcomes Study adaptada para o português no Estudo Pró-Saúde. Cad Saude Publica. 2005;21(3):703-14. http://dx.doi.org/10.1590/S0102-311X2005000300004. PMid:15868028.

19. Chor D, Griep RH, Lopes CS, Faerstein E. Medidas de rede e apoio social no Estudo Pró-Saúde: prétestes e estudo piloto. Cad Saude Publica. 2001;17(4):887-96. http://dx.doi.org/10.1590/S0102 311X2001000400022. PMid:11514869.

20. Segall-Corrêa AM, Marin-León L, Melgar-Quiñonez H, Pérez-Escamilla R. Refinement of the Brazilian Household Food Insecurity Measurement Scale: Recommendation for a 14-item EBIA. Rev Nutr. 2014;27(2):41-51. http://dx.doi.org/10.1590/1415-52732014000200010.

21. Brasil. Ministério da Saúde. Universidade do Estado do Rio de Janeiro. A creche como promotora da amamentação e da alimentação adequada e saudável: livreto para os gestores [Internet]. Brasília: Editora MS; 2018 [citado em 2019 fev 12]. Disponível em: http://189.28.128.100/dab/docs/portaldab/ publicacoes/a_creche_promotora_amamentacao_livreto_gestores.pdf

22. Brasil. Ministério da Saúde. Pesquisa Nacional de Demografia e Saúde da Criança e da Mulher - PNDS 2006: dimensões do processo reprodutivo e da saúde da criança [Internet]. Brasília: Editora MS; 2009 [citado em 2019 fev 12]. Disponível em: http://bvsms.saude.gov.br/bvs/publicacoes/pnds_crianca_mulher.pdf

23. Coelho LC, Asakura L, Sachs A, Erbert I, Novaes CRL, Gimeno SGA. Food and Nutrition Surveillance System/SISVAN: getting to know the feeding habits of infants under 24 months of age. Cien Saude Colet. 2015;20(3):727-38. http://dx.doi.org/10.1590/1413-81232015203.15952014. PMid:25760113.

24. Victora CG, Bahl R, Barros AJD, França GVA, Horton S, Krasevec J, et al. Breastfeeding in the $21 \mathrm{st}$ century: epidemiology, mechanisms, and lifelong effect. Lancet. 2016;387(10017):475-90. http://dx.doi. org/10.1016/S0140-6736(15)01024-7. PMid:26869575.

25. Pereira-Santos M, Sousa SM, Santana OD, Nepomuceno RA Fo, Lisboa CS, Almeida LMR, et al. Prevalência e fatores associados à interrupção precoce do aleitamento materno exclusivo: metanálise de estudos epidemiológicos brasileiros. Rev Bras Saúde Mater Infant. 2017;17(1):69-78. http://dx.doi. org/10.1590/1806-93042017000100004.

26. World Health Organization. Sixty-fifth World Health Assembly: resolutions and decisions. Geneva: WHO 2012.

27. Venancio SI, Giugliani ER, Silva OL, Stefanello J, Benicio MH, Reis MC, et al. Associação entre o grau de implantação da Rede Amamenta Brasil e indicadores de amamentação. Cad Saude Publica. 2016;32(3):e00010315. http://dx.doi.org/10.1590/0102-311X00010315. PMid:27027452.

28. Bortolini GA, Vitolo MR, Gubert MB, Santos LMP. Iniquidades sociais influenciam a qualidade e a diversidade da dieta de crianças brasileiras de 6 a 36 meses. Cad Saude Publica. 2015;31(11):2413-24. http://dx.doi. org/10.1590/0102-311X00153414. PMid:26840820.

29. Demmer E, Cifelli CJ, Houchins J, Fulgoni VL 3rd. The pattern of complementary foods in american infants and children aged 0-5 years old-a cross-sectional analysis of data from the NHANES 2011-2014. Nutrients. 2018;10(7):827. http://dx.doi.org/10.3390/nu10070827. PMid:29949890. 
30. Alves MN, Muniz LC, Vieira MFA. Consumo alimentar entre crianças brasileiras de dois a cinco anos de idade: Pesquisa Nacional de Demografia e Saúde (PNDS), 2006. Cien Saude Colet. 2013;18(11):3369-77. http://dx.doi.org/10.1590/S1413-81232013001100026. PMid:24196901.

31. Machado RHV, Feferbaum R, Leone C. Consumo de frutas no Brasil e prevalência de obesidade. J Hum Growth Dev. 2016;26(2):243-52. http://dx.doi.org/10.7322/jhgd.119293.

32. Jaime PC, Frias PG, Monteiro HOC, Almeida PVB, Malta DC. Healthcare and unhealthy eating among children aged under two years: data from the National Health Survey, Brazil, 2013. Rev Bras Saúde Mater Infant. 2016;16(2):149-57. http://dx.doi.org/10.1590/1806-93042016000200005.

33. Giesta JM, Zoche E, Corrêa RS, Bosa VL. Fatores associados à introdução precoce de alimentos ultraprocessados na alimentação de crianças menores de dois anos. Cien Saude Colet. 2019;24(7):238797. http://dx.doi.org/10.1590/1413-81232018247.24162017. PMid:31340258.

34. Langellier BA, Pia Chaparro M, Whaley SE. Social and institutional factors that affect breastfeeding duration among WIC participants in Los Angeles County, California. Matern Child Health J. 2012;16(9):1887-95. http://dx.doi.org/10.1007/s10995-011-0937-z. PMid:22205423.

35. Souza MHN, Sodré VRD, Silva FNF. Prevalência e fatores associados à prática da amamentação de crianças que frequentam uma creche comunitária. Cienc Enferm. 2015;21(1):55-67. http://dx.doi.org/10.4067/ S0717-95532015000100006.

36. Santana GS, Giugliani ERJ, Vieira TO, Vieira GO. Fatores associados à manutenção da amamentação por 12 meses ou mais: revisão sistemática. J Pediatr. 2018;94(2):104-22. http://dx.doi.org/10.1016/j. jped.2017.06.013. PMid:28886401.

37. Wenzel D, Souza SB. Fatores associados ao aleitamento materno nas diferentes Regiões do Brasil. Rev Bras Saúde Mater Infant. 2014;14(3):241-9. http://dx.doi.org/10.1590/S1519-38292014000300005.

38. Cotta RMM, Machado JC. Programa Bolsa Família e segurança alimentar e nutricional no Brasil: revisão crítica da literatura. Rev Panam Salud Publica. 2013;33(1):54-60. http://dx.doi.org/10.1590/S102049892013000100008. PMid:23440158.

39. Sperandio N, Rodrigues CT, Franceschini SCC, Priore SL. Impacto do Programa Bolsa Família no consumo de alimentos: estudo comparativo das regiões Sudeste e Nordeste do Brasil. Cien Saude Colet. 2017;22(6):1771-80. http://dx.doi.org/10.1590/1413-81232017226.25852016. PMid:28614498.

40. Martins MC, Aires JS, Dantas KB, Sabino LMM, Alves MDS, Ximenes LB. Consumo alimentar de famílias de pré-escolares em situação de (in)segurança alimentar. Cienc Enferm. 2015;21(3):63-71. http://dx.doi. org/10.4067/S0717-95532015000300006.

41. Rocha NP, Milagres LC, Novaes JF, Franceschini SCC. Associação de insegurança alimentar e nutricional com fatores de risco cardiometabólicos na infância e adolescência: uma revisão sistemática. Rev Paul Pediatr. 2016;34(2):225-33. http://dx.doi.org/10.1016/j.rpped.2015.08.007. PMid:26564327.

42. Oddo VM, Ickes SB. Maternal employment in low- and middle-income countries is associated with improved infant and young child feeding. Am J Clin Nutr. 2018;107(3):335-44. http://dx.doi.org/10.1093/ ajcn/nqy001. PMid:29566201.

43. Oliveira MIC, Rigotti RR, Boccolini CS. Fatores associados à falta de diversidade alimentar no segundo semestre de vida. Cad Saude Colet. 2017;25(1):65-72. http://dx.doi.org/10.1590/1414-462x201700010204.

44. Vieira DAS, Castro MA, Fisberg M, Fisberg RM. Qualidade nutricional dos padrões alimentares de crianças: existem diferenças dentro e fora da escola? J Pedriatr. 2017;93(1):47-57. http://dx.doi.org/10.1016/j. jped.2016.03.008. PMid:27362785.

45. Figueroa Pedraza D, Queiroz D, Gama JS. Avaliação do consumo alimentar de crianças brasileiras assistidas em creches: uma revisão sistemática. Rev Bras Saúde Mater Infant. 2015;15(1):17-31. http://dx.doi. org/10.1590/S1519-38292015000100002.

46. Wolf MR, Barros AA Fo. Estado nutricional dos beneficiários do Programa Bolsa Família no Brasil: uma revisão sistemática. Cien Saude Colet. 2014;19(5):1331-8. http://dx.doi.org/10.1590/1413-81232014195.05052013. PMid:24897198.

47. Schincaglia RM, Oliveira AC, Sousa LM, Martins KA. Práticas alimentares e fatores associados à introdução precoce da alimentação complementar entre crianças menores de seis meses na região noroeste de Goiânia. Epidemiol Serv Saude. 2015;24(3):465-74. http://dx.doi.org/10.5123/S1679-49742015000300012.

48. Corrêa RS, Vencato PH, Rockett FC, Bosa VL. Padrões alimentares de escolares: existem diferenças entre crianças e adolescentes? Cien Saude Colet. 2017;22(2):553-62. http://dx.doi.org/10.1590/141381232017222.09422016 . 